\title{
Implementation of a Reference-Scaled Average Bioequivalence Approach for Highly Variable Acetylsalicylic Acid in Fixed-Dose Combination with Clopidogrel Versus Enteric Aspirin in Chinese Subjects Under Fasting Conditions: A Phase 1, Open- Label, Randomized, Crossover Study
}

Lu Wang · Yujing Di · Tingting Guo · Jeffrey E. Ming · Fangyuan Kong · Huiqiu Yin •

Linlin Zhang $\cdot$ Fang Xie $\cdot \mathrm{Na}$ Yang $\cdot$ Chuan Ping $\cdot$ Yi Li $\cdot$ Jie Hou

Received: March 31, 2020 / Published online: May 16, 2020

(C) The Author(s) 2020

\section{ABSTRACT}

Introduction: Dual antiplatelet therapy, aspirin and a $\mathrm{P}_{2} \mathrm{Y}_{12}$ inhibitor, is recommended to prevent thrombotic complications of acute coronary syndrome. Clopidogrel plus acetylsalicylic acid combination is the most commonly used dual antiplatelet therapy recommended by international guidelines and in Chinese clinical practice. Poor adherence to dual antiplatelet therapy or premature interruption of dual antiplatelet therapy is an important contributor to

Digital Features To view digital features for this article go to https://doi.org/10.6084/m9.figshare.12206147.

Electronic supplementary material The online version of this article (https://doi.org/10.1007/s12325$020-01369-z)$ contains supplementary material, which is available to authorized users.

L. Wang · Y. Di · T. Guo · J. Hou (ه)

Phase I Center, Luzhong Hospital, Shandong, China

e-mail: jie.hou@estartpoc.com

J. E. Ming

Research and Development, Sanofi, New York, USA

F. Kong · N. Yang $\cdot$ C. Ping · Y. Li

Research and Development, Sanofi, Beijing, China

H. Yin · F. Xie

Medical, Sanofi, Shanghai, China

L. Zhang

Medical, Sanofi, Beijing, China cardiovascular mortality and lethal cardiovascular events. Clopidogrel + acetylsalicylic acid fixed-dose combination enhances adherence to dual antiplatelet therapy. Herein, we aimed to evaluate bioequivalence of acetylsalicylic acid and clopidogrel in fixed-dose combination compared with simultaneous administration of their individual formulations in healthy Chinese subjects under fasting conditions.

Methods: This was a randomized, single-center, open-label, three-sequence, three-period, twotreatment, crossover study with a washout period of 10 days conducted in healthy Chinese volunteers. Subjects were randomized to receive CoPlavix $^{\circledR}$ (test formulation- fixed-dose combination of $100 \mathrm{mg}$ acetylsalicylic acid and $75 \mathrm{mg}$ clopidogrel) once and reference formulations (coadministration of individual formulations of $100 \mathrm{mg}$ acetylsalicylic acid and $75 \mathrm{mg}$ clopidogrel) twice during the study period. Pharmacokinetic parameters were analyzed for acetylsalicylic acid, its metabolite salicylic acid, clopidogrel, and its metabolite SR26334. As acetylsalicylic acid shows high intrasubject variability, the referencescaled average bioequivalence (RSABE) approach was implemented for acetylsalicylic acid analysis, while bioequivalence of clopidogrel was assessed using the average bioequivalence method. Point ratios and confidence intervals (CIs) for AUC, $\mathrm{AUC}_{\text {last, }}$ and $C_{\max }$ for acetylsalicylic acid and clopidogrel were calculated. 
Results: In total, 171 healthy subjects were enrolled in this study. Subjects were randomized and 170 subjects were treated with test or reference formulation; 164 subjects completed the study. Regarding acetylsalicylic acid exposure, as reference within-subject standard deviation $\left(\mathrm{SD}_{\mathrm{W}}\right)$ was at least 0.294 for acetylsalicylic acid $C_{\text {max }}, \mathrm{AUC}_{\text {last }}$, and AUC, the RSABE analysis method was used to assess bioequivalence for all three parameters. The point estimates were within the $0.80-1.25$ range $(1.19,1.09$, and 1.04, respectively), and upper one-sided 95\% CIs of scaled average bioequivalence metric were at most $0(-0.30,-0.14$, and -0.10 , respectively). Thus, bioequivalence was demonstrated with acetylsalicylic acid. Bioequivalence was also achieved with clopidogrel as the $90 \%$ CIs for geometric mean ratios of clopidogrel $C_{\text {max }}, \mathrm{AUC}_{\text {last }}$, and AUC were within the bioequivalence range (0.80-1.25).

Conclusion: Application of the reference-scaled average bioequivalence approach to evaluate bioequivalence of acetylsalicylic acid in Chinese male and female healthy volunteers under fasting conditions demonstrated bioequivalence of test and reference formulations.

Trial Registration: CTR20181695.

Keywords: Acetylsalicylic acid; Bioequivalence; Clopidogrel; Co-Plavix; Fixed-dose combination; RSABE

\section{Key Summary Points}

Why carry out this study?

Poor adherence to dual antiplatelet therapy or its premature interruption is an important contributor to cardiovascular mortality.

Bioequivalence of the fixed-dose combination compared to simultaneous intake of single drugs in the Chinese population is unknown.

Reference-scaled average bioequivalence approach could be used to establish bioequivalence of drugs with high intrasubject variability.

\section{What was learned from the study?}

Fixed-dose combination of acetylsalicylic acid and clopidogrel is bioequivalent to the individual formulations in healthy Chinese patients.

Exposure of subjects to acetylsalicylic acid in fixed-dose combination demonstrated bioequivalence using the reference-scaled average bioequivalence method as the point estimates were within the $0.80-1.25$ range, and the upper one-sided 95\% CIs of scaled average bioequivalence metric were no greater than 0 .

Bioequivalence was also achieved with clopidogrel as the $90 \%$ CIs for geometric mean ratios of clopidogrel $C_{\text {max }}, \mathrm{AUC}_{\text {last }}$, and AUC were within the bioequivalence range $(0.80-1.25$.

\section{INTRODUCTION}

Dual antiplatelet therapy with acetylsalicylic acid and a $\mathrm{P}_{2} \mathrm{Y}_{12}$ adenosine diphosphate (ADP) receptor antagonist, such as clopidogrel, plays an important role in the standard of care treatment for acute coronary syndrome [1]. Acetylsalicylic acid irreversibly inhibits platelet cyclooxygenase, leading to the inhibition of thromboxane A2 which is an inducer of platelet aggregation and vasoconstriction [2]. Clopidogrel inhibits ADP-induced platelet aggregation via formation of an inactive carboxylic acid and an active thiol metabolite [3]. Despite various clinical guideline recommendations, there is a significant gap between these guidelines and clinical practice because of issues with drug compliance and premature interruption/disruption of dual antiplatelet therapy, leading to cardiovascular mortality and lethal cardiovascular events [4-6]. Clopidogrel and acetylsalicylic acid enteric-coated (EC) fixed-dose combination provides a pharmaceutical option to ensure better adherence and compliance of dual antiplatelet therapy.

The fixed-dose combination was developed to target patients who already have been 
receiving both clopidogrel and acetylsalicylic acid for the prevention of atherothrombotic events. Three phase III studies (CURE, CLARITY, COMMIT) [7-10] have been conducted in patients with acute coronary syndrome (including Chinese patients) who received clopidogrel in addition to aspirin worldwide, and its effectiveness and safety have been fully demonstrated. However, the bioequivalence of the fixed-dose combination compared to simultaneous intake of single drugs in the Chinese population was unknown, hence this study was conducted to assess bioequivalence of the fixed-dose combination in the Chinese population.

\section{METHODS}

The study protocol and informed consent to participate were reviewed and approved by the Institutional Review Board of Peking University Care Luzhong Hospital. The study was conducted in accordance with consensus ethics principles derived from international ethics guidelines, including the Declaration of Helsinki and The International Council for Harmonisation of Technical Requirements for Pharmaceuticals for Human Use guidelines for Good Clinical Practice, and all applicable laws, rules, and regulations.

\section{Study Design}

A randomized, single-center, open-label, twotreatment, three-period, three-sequence, crossover study (study number CTR20181695) with a 10-day washout period between administrations was conducted in healthy Chinese subjects at Peking University (PKU) Care Luzhong Hospital, China, from October 15, 2018, to December 11, 2018. The investigational medical product was Co-Plavix ${ }^{\circledR}$ (test), a fixed-dose combination of $75 \mathrm{mg}$ clopidogrel and $100 \mathrm{mg}$ acetylsalicylic acid, which was tested against the coadministration of Bayaspirin ${ }^{\circledR}$ (EC, $100 \mathrm{mg}$ acetylsalicylic acid) and Plavix ${ }^{\circledR}$ (75 mg clopidogrel), constituting the reference formulations.

Of 171 subjects who fulfilled the inclusion and exclusion criteria, 171 subjects were randomized to any one of the three sequences [test (T)/reference (R)/R, R/T/R, R/R/T] (Fig. 1) and were provided with a relevant drug number after the screening period using the RANDOM 2.1 system. The randomization scheme consisted of a block size of 6 , with 171 subjects divided into two treatment groups with three periods and three sequences. A total of 170 subjects were treated with test or reference formulation. All treatments were administered orally after overnight fasting for at least $10 \mathrm{~h}$ before administration of the test and reference drug regimens. The test group (T) received one Co-Plavix ${ }^{\circledR}$ tablet and the reference group (R) received simultaneous administration of Bayaspirin ${ }^{\circledR}$ and Plavix ${ }^{\circledR}$ as individual formulations. In each period, only a single dose of the combination tablet of the individual formulations was administered. The subjects were allowed to have standard lunch and dinner at least $4 \mathrm{~h}$ and $10 \mathrm{~h}$ post administration. The subjects were also followed up for 5-7 days after the last administration.

\section{Subjects}

Healthy Chinese male and female subjects with age at least 18 years, body weight between 50.0 and $95.0 \mathrm{~kg}$ and 45.0 and $90.0 \mathrm{~kg}$ for men and women, respectively, and body mass index (BMI) between 18.5 and $27.9 \mathrm{~kg} / \mathrm{m}^{2}$ were included. All subjects were certified to be healthy by a comprehensive health assessment that included electrocardiogram (ECG), vital signs, blood biochemical examination, and other laboratory parameters. Female subjects of childbearing age were required to have negative results on a pregnancy test and only those who agreed to use an appropriate method of contraception during the study period were included. The enrolled subjects were not allowed to consume citrus fruits or their juices within 5 days before initiation of the study. Subjects with any of the following characteristics were excluded from the study: clinical/pathological signs, frequent nausea, headache and/or migraine, alcohol (more than $40 \mathrm{~g}$ /day) or drug abuse, smoking (more than 5 cigarettes/day), excessive consumption of caffeine beverages 


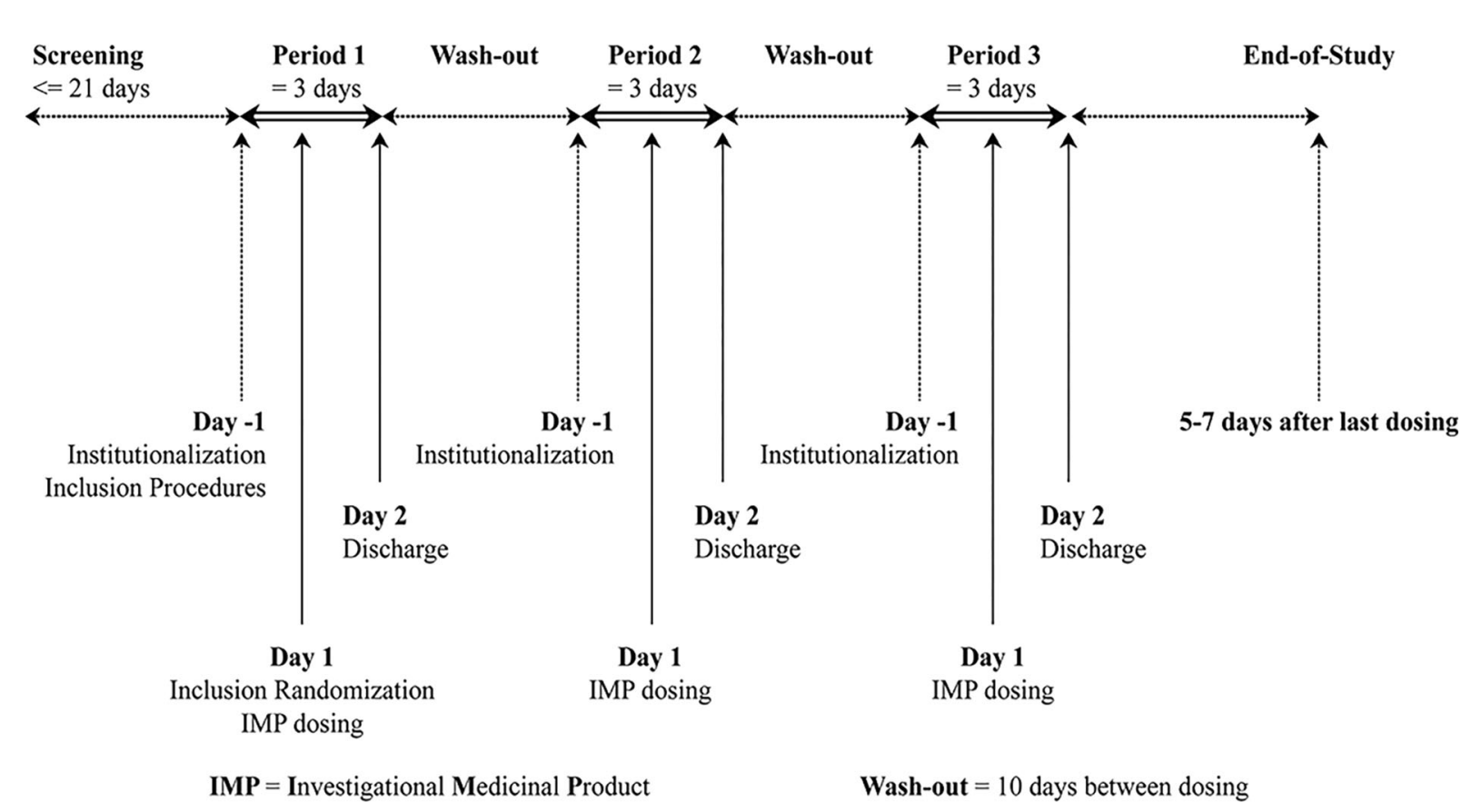

Fig. 1 Study design for the bioequivalence evaluation of test and reference

(more than 4 cups/day), donated blood within 3 months before inclusion, usage of any medication (including traditional Chinese medicine or herbal supplements) within 14 days before inclusion, any vaccination within 28 days before inclusion, and administration of any biologics within 4 months before inclusion.

\section{Outcomes and End Points}

For acetylsalicylic acid, bioequivalence between the two formulations of acetylsalicylic acid was assessed using the mixed scaled average bioequivalence scale approach for $C_{\max }$, $\mathrm{AUC}_{\text {last}}$, and AUC. For each log-transformed parameter, a linear mixed-effects model was fitted, allowing for treatment-specific within-subject variance terms and the estimated value of the withinsubject standard deviation $\left(\mathrm{SD}_{\mathrm{W}}\right)$ for the reference formulation was obtained. If $\mathrm{SD}_{\mathrm{W}}$ is less than 0.294 for $C_{\max }, \mathrm{AUC}_{\text {last}}$, or AUC of acetylsalicylic acid, then the traditional standard average bioequivalence scale approach was used where bioequivalence was concluded if the $90 \%$ $\mathrm{CI}$ for the formulation ratio (test/reference) was within the range of $0.80-1.25$. If $\mathrm{SD}_{\mathrm{W}}$ is at least 0.294 for $C_{\max }, \mathrm{AUC}_{\text {last}}$, or $\mathrm{AUC}$ of acetylsalicylic acid, then the reference-scaled average bioequivalence analysis described by Haidar et al. [11] for highly variable drugs was conducted (i.e., upper one-sided 95\% CI for the scaled average bioequivalence scale metrics). Bioequivalence was concluded if the point estimate (test/reference geometric mean ratio) was within $0.80-1.25$ and the upper one-sided 95\% confidence limit for scaled average bioequivalence scale metric $\left(\mu_{\mathrm{T}}-\mu_{\mathrm{R}}\right)^{2}-\theta \sigma_{\mathrm{WR}}^{2}$ was at most 0 where $\theta=(\ln \Delta)^{2} / \sigma_{\mathrm{w} 0}^{2}$ and $\Delta=1.25$, the usual average bioequivalence scale upper limit for the untransformed test/reference ratio of geometric means, and $\sigma_{\mathrm{w} 0}=0.25$.

For clopidogrel, the differences in log-transformed $C_{\text {max }}, \mathrm{AUC}$, and $\mathrm{AUC}_{\text {last }}$ were assessed using a linear mixed-effect model with fixed terms for sequence, period, and formulation, random terms for subjects within sequence, and a factor-analytic variance-covariance structure using the FAO(2) option, grouped by formulation for subject within sequence using SAS Proc Mixed $^{\circledR}$. Point estimates and 90\% CIs for the geometric means ratio of $C_{\max }, \mathrm{AUC}$, and AUC $_{\text {last }}$ between 2 formulations were obtained within this mixed-effect model framework and then converted to the ratio scale by antilog 
transformation. Bioequivalence was concluded if the $90 \%$ CIs for the ratio were entirely within [0.80-1.25].

Adverse events were coded according to the Medical Dictionary for Regulatory Activities (MedDRA, version currently in use by Sanofi at the time of database lock).

\section{Pharmacokinetic Evaluation}

Pharmacokinetic parameters were measured by a bioanalytical method using validated liquid chromatography-tandem mass spectrometry (LC-MS/MS). Bioanalysis was performed as per the good laboratory practice (GLP) requirements identified in the Organization for Economic Co-operation and Development principles of GLP (as revised in 1997), ENV/MC/ CHEM (98)17, and the GLP regulations applicable to the local country. Clopidogrel, SR26334, acetylsalicylic acid, and salicylic acid were the analytes estimated by LC-MS/MS. The estimation was performed by Covance, China. Clopidogrel, acetylsalicylic acid, and salicylic acid concentrations were measured and analyzed by pretreating their plasma samples with liquid-liquid extraction, whereas for SR26334, the plasma samples were pretreated with protein precipitation. The lower limits of quantification (LLOQs) were $5 \mathrm{pg} / \mathrm{mL}, 5 \mathrm{ng} / \mathrm{mL}, 5 \mathrm{ng} /$ $\mathrm{mL}$, and $100 \mathrm{ng} / \mathrm{mL}$ for clopidogrel, SR26334, acetylsalicylic acid and salicylic acid, respectively, and the assays had adequate accuracy and precision in calibration curves ranging from 5.00 to $5000 \mathrm{pg} / \mathrm{mL}$ (clopidogrel), 5.00 to $5000 \mathrm{ng} / \mathrm{mL}$ (SR26334 and acetylsalicylic acid), and 100 to $10,000 \mathrm{ng} / \mathrm{mL}$ (salicylic acid). Blood samples for the determination of plasma acetylsalicylic acid and salicylic acid concentrations were collected at pre-dose and 2, 3, 3.5, $4,4.5,5,5.5,6,6.5,7,7.5,8,10,12$, and $16 \mathrm{~h}$ post-dose in each treatment period. Blood samples for the determination of plasma clopidogrel and SR26334 concentrations were collected at pre-dose, 10, 20, 30, and 45 min postdose, and 1, 1.5, 2, 3, 4, 6, 8, 12, 16, and $24 \mathrm{~h}$ post-dose in each treatment period. The pharmacokinetic parameters of the plasma samples of acetylsalicylic acid, salicylic acid, clopidogrel, and SR26334 were measured by noncompartmental method using WinNonlin software. The primary pharmacokinetic parameters were $C_{\text {max }}$ AUC, and $\mathrm{AUC}_{\text {last }}$ for clopidogrel and acetylsalicylic acid, whereas the other parameters analyzed were $T_{\max }$ and $t_{1 / 2 z}$ for clopidogrel and acetylsalicylic acid. The definitions and mode of determination of pharmacokinetic parameters are provided in Table S1 in the supplementary material.

\section{Safety Assessment}

All 170 randomized subjects were included in the safety assessment that included clinical laboratory parameters (hematology, biochemistry, urinalysis, coagulation), physical examination, vital signs, and standard 12-lead ECGs. Adverse events (AEs) and treatment-emergent adverse events (TEAEs) were graded as per the National Cancer Institute-Common Terminology Criteria for Adverse Events (CTCAE) $\mathrm{v} 4.03$ and were monitored throughout the study period.

\section{Sample Size Determination}

Up to 171 subjects were enrolled to have approximately 135 subjects for completion, which was deemed sufficient to provide a $90 \%$ overall power for pharmacokinetic evaluation.

The calculation of sample size for this study was based on the completed bioavailability and bioequivalence studies (two published and one unpublished study) [12-14] conducted at Sanofi for the fixed-dose combinations of clopidogrel and enteric acetylsalicylic acid compared with the reference formulations (Plavix ${ }^{\circledR}$ and Bayaspirin $\left.{ }^{\circledR}\right)$, which were conducted in healthy Japanese and Korean male subjects. The $\mathrm{SD}_{\mathrm{W}}$ for clopidogrel was estimated from studies (one published and one unpublished study) $[12,14]$ and that for acetylsalicylic acid was estimated from studies (two published and one unpublished study) [12-14]. For clopidogrel, the pooled point estimates of the pharmacokinetic parameters ratios were $0.99,0.98$, and 1.10 ; the estimates of $\mathrm{SD}_{\mathrm{W}}$ (on the natural log scale) were $0.344,0.335$, and 0.365 for $\mathrm{AUC}_{\text {last}}$, AUC, and 
$C_{\text {max }}$, respectively. For acetylsalicylic acid, the pooled point estimates of pharmacokinetics parameters ratios were $1.10,1.10$, and 1.08 ; the estimates of $\mathrm{SD}_{\mathrm{W}}$ (on the natural log scale) were $0.488,0.416$, and 0.696 for $\mathrm{AUC}_{\text {last }}$ AUC, and $C_{\text {max }}$, respectively (the true ratios and true $\mathrm{SD}_{\mathrm{W}}$ for sample size calculation are presented in Table S2 in the supplementary material).

The power of concluding bioequivalence with 135 subjects is presented in Table S2. For acetylsalicylic acid, the scaled average bioequivalence scale approach is used, while the standard average bioequivalence scale approach was applied for clopidogrel. The overall power was calculated as a function of the lowest power in clopidogrel and the lowest power in acetylsalicylic acid because AUC and $C_{\max }$ were assumed to be highly correlated parameters; therefore, no power loss between AUC and $C_{\max }$ of the same ingredient was considered in the sample size calculation. So the overall power was $90.24 \%(94 \% \times 96 \%)$, which was higher than $90 \%$. In summary, a total of 135 subjects achieved an overall $90 \%$ power to conclude bioequivalence between formulations (fixeddose combination vs individual formulation) for clopidogrel and acetylsalicylic acid. Allowing for a $20 \%$ dropout rate, the total number of subjects to enroll was 171 .

\section{Statistical Analysis}

Pharmacokinetic parameters of clopidogrel, acetylsalicylic acid, and their respective major metabolites SR26334 and salicylic acid were summarized using descriptive statistics. Bioequivalence between the fixed-dose combination and individual formulations was assessed using the mixed scaled average bioequivalence scale approach for $C_{\max }, \mathrm{AUC}_{\text {last}}$, and AUC. $C_{\max }$, $\mathrm{AUC}_{\text {last, }}$ and $\mathrm{AUC}$ were log-transformed and were fitted into a linear mixed-effects model allowing for treatment-specific within-subject variance terms, and the estimated value of the $\mathrm{SD}_{\mathrm{W}}$ for reference formulation was obtained. If this value was less than 0.294 , the traditional average bioequivalence scale analysis was performed within the mixed model framework (i.e., point estimate and $90 \%$ CI for the ratio of geometric means for the two formulations). If the value was at least 0.294 , the reference-scaled average bioequivalence analysis described by Haidar et al. [11] was performed (i.e., upper onesided 95\% CI for the scaled average bioequivalence scale metrics). For clopidogrel exposure, average bioequivalence scale analysis was performed on the log-transformed pharmacokinetic parameters. All statistical calculations were performed using SAS. Bioequivalence was concluded if the point estimate (test/reference geometric mean ratio) was within the range of 0.80-1.25 and the upper one-sided 95\% CI for the scaled average bioequivalence scale metric was defined as: $\left(\mu_{\mathrm{T}}-\mu_{\mathrm{R}}\right)^{2}-\theta \quad \sigma_{\mathrm{WR}}^{2} \leq 0$ where $\theta=(\ln \Delta)^{2} / \sigma_{\mathrm{w} 0}^{2}$ and $\Delta=1.25$, the usual average bioequivalence upper limit for the untransformed test/reference ratio of geometric means, and $\sigma_{\mathrm{w} 0}=0.25$ where $\mu_{\mathrm{T}}$ is the population average response of the log-transformed measure for the test (T) formulation, $\mu_{\mathrm{R}}$ is the population average response of the log-transformed measure for the reference (R) formulation, and $\sigma_{\mathrm{WR}}^{2}$ is the population within-subject variance of the reference formulation.

The safety evaluation was based on the review of the individual laboratory values (clinically significant abnormalities) provided in descriptive statistics (summary tables, graphics).

\section{RESULTS}

\section{Subject Demographics}

Among the 170 treated subjects, the mean age was 35.8 years, $55.3 \%$ were male, and the mean BMI was $23.43 \mathrm{~kg} / \mathrm{m}^{2}$. None of the subjects received any concomitant medication during the study period (Table 1 ). The subjects were randomized into three sequences: $\mathrm{T} / \mathrm{R} / \mathrm{R}$ $(n=58), \mathrm{R} / \mathrm{T} / \mathrm{R}(n=57)$, and R/R/T $(n=55)$. Six subjects discontinued from the study because of post-treatment AEs (one subject), personal reasons (three subjects), and lost to follow-up (two subjects). 
Table 1 Baseline characteristics

\begin{tabular}{ll}
\hline Variables & $\begin{array}{l}\text { Number of subjects } \\
\text { after treatment } \\
(\boldsymbol{N}=\mathbf{1 7 0})\end{array}$ \\
\hline Age (years, mean $\left.\pm \mathrm{SD}^{\mathrm{a}}\right)$ & $35.8 \pm 9.6$ \\
Male $[n(\%)]$ & $94(55.3 \%)$ \\
Weight $(\mathrm{kg}$, mean $\pm \mathrm{SD})$ & $64.85 \pm 9.73$ \\
Height $(\mathrm{cm}$, mean $\pm \mathrm{SD})$ & $166.1 \pm 8.5$ \\
$\mathrm{BMI}^{\mathrm{b}}\left(\mathrm{kg} / \mathrm{m}^{2}\right.$, mean $\left.\pm \mathrm{SD}\right)$ & $23.43 \pm 2.34$ \\
\hline
\end{tabular}

a Standard deviation

b Body mass index

\section{Pharmacokinetic Evaluation}

The mean plasma concentration-time profiles of acetylsalicylic acid and clopidogrel are presented in Figs. 2 and 3. The pharmacokinetic parameters are provided in Table 2.

Acetylsalicylic acid: Following the administration of fixed-dose combination or Bayaspirin, the systemic exposure to acetylsalicylic acid, based on $C_{\text {max }}, \mathrm{AUC}_{\text {last }}$, and $\mathrm{AUC}$, was similar between the two formulations, with respective arithmetic mean values of $790 \mathrm{ng} / \mathrm{mL}, 967 \mathrm{ng} \mathrm{h} /$ $\mathrm{mL}$, and $1020 \mathrm{ng} \mathrm{h} / \mathrm{mL}$ for fixed-dose combination and $688 \mathrm{ng} / \mathrm{mL}, \quad 901 \mathrm{ng} \mathrm{h} / \mathrm{mL}$, and $986 \mathrm{ng} \mathrm{h} / \mathrm{mL}$ for Bayaspirin. Acetylsalicylic acid reached the maximum plasma concentration in 5.00 and $5.50 \mathrm{~h}$ (median) post-dose for fixeddose combination and Bayaspirin, respectively. The mean elimination half-lives of acetylsalicylic acid were 0.455 and $0.471 \mathrm{~h}$ for fixed-dose combination and Bayaspirin, respectively.

Clopidogrel: The systemic exposure to clopidogrel, based on $C_{\max }, \mathrm{AUC}_{\text {last }}$, and AUC, was similar between the two formulations, with respective arithmetic mean values of $2390 \mathrm{pg} /$ $\mathrm{mL}, 2810 \mathrm{pg} \mathrm{h} / \mathrm{mL}$, and $3020 \mathrm{pg} \mathrm{h} / \mathrm{mL}$ for fixeddose combination and $2130 \mathrm{pg} / \mathrm{mL}, 2980 \mathrm{pg} \mathrm{h} /$ $\mathrm{mL}$, and $3240 \mathrm{pg} \mathrm{h} / \mathrm{mL}$ for clopidogrel. Clopidogrel reached the maximum plasma concentration in 0.50 and $0.75 \mathrm{~h}$ (median) post-dose for fixed-dose combination and Plavix ${ }^{\circledR}$, respectively. The elimination half-lives of clopidogrel after administration of fixed-dose combination and Plavix ${ }^{\circledR} 75 \mathrm{mg}$ were approximately 4.94 and $5.88 \mathrm{~h}$, respectively.
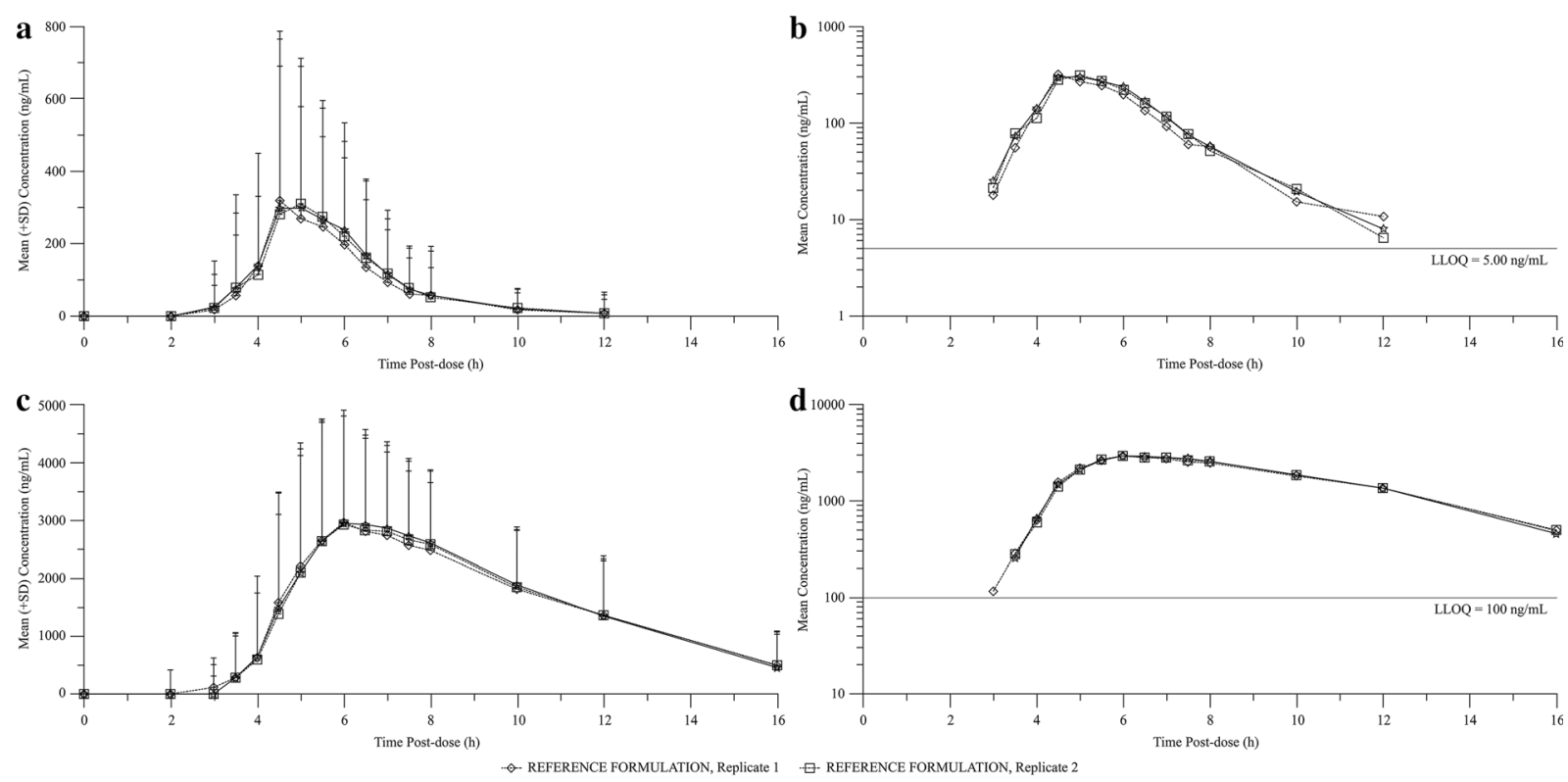

Fig. 2 Mean plasma concentration versus time profiles of acetylsalicylic acid and salicylic acid following the administration of a single oral dose of test and reference (replicates 1 and 2) formulations to healthy Chinese

subjects. a Linear model-acetylsalicylic acid; b semilogarithmic scale-acetylsalicylic acid; c linear modelsalicylic acid; d semi-logarithmic scale-salicylic acid 

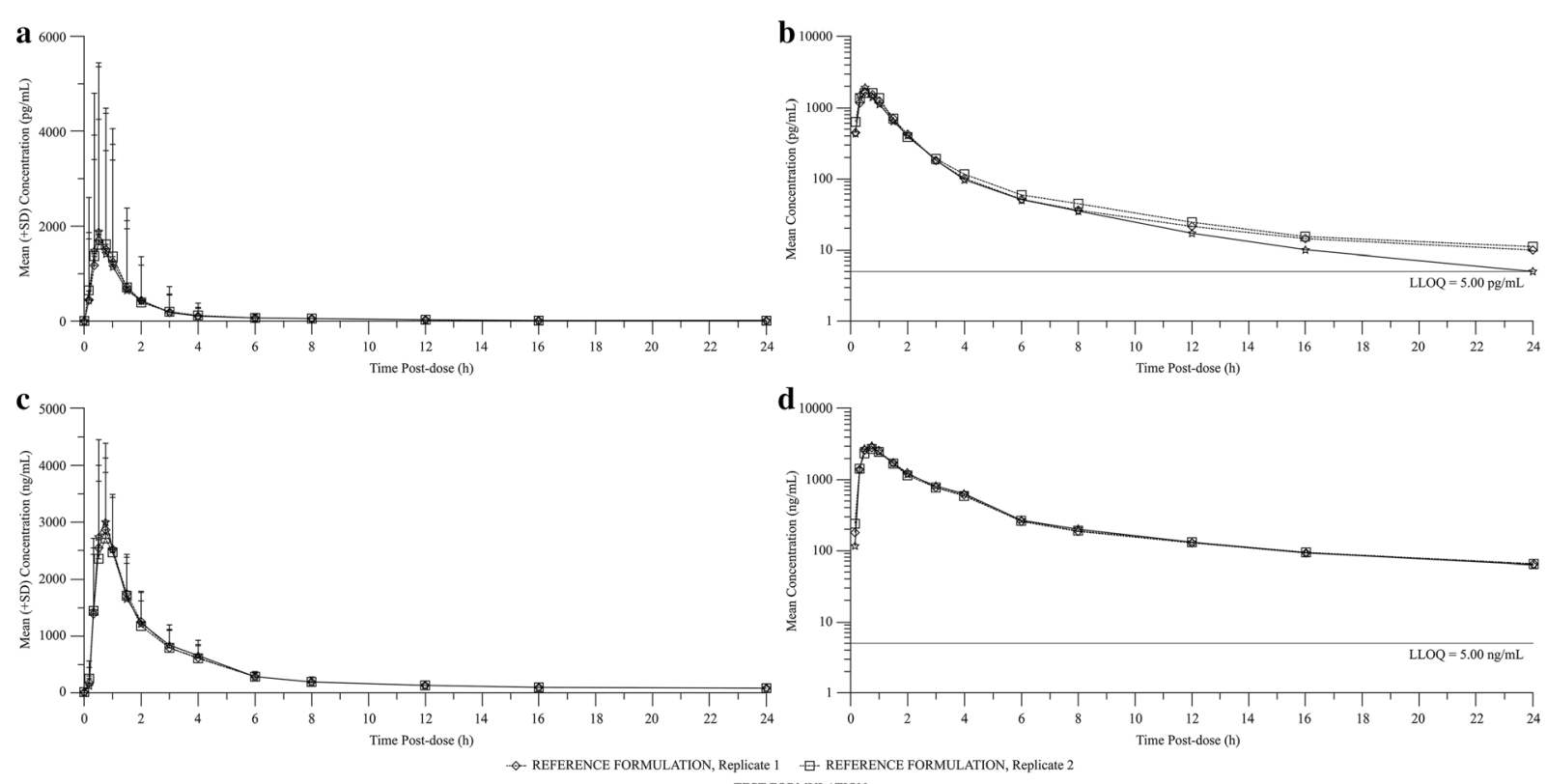

Fig. 3 Mean plasma concentration versus time profiles of clopidogrel and SR26334 after single oral dose of test and reference (replicates 1 and 2) formulations to healthy

\section{Bioequivalence Evaluation}

Considering that the within-subject variability for the reference $\left(S_{\mathrm{WR}}\right)$ for log-transformed $C_{\text {max }}, \mathrm{AUC}_{\text {last, }}$ and $\mathrm{AUC}$ was at least 0.294 for acetylsalicylic acid, the mixed reference-scaled average bioequivalence analysis was performed to assess bioequivalence (Table 3 ). The point estimate ratios and 95\% CIs calculated for AUC, AUC $_{\text {last, }}$ and $C_{\max }$ are summarized in Table 4. For the ratios comparing $C_{\max }, \mathrm{AUC}_{\text {last}}$, and AUC for acetylsalicylic acid following administration of the test versus reference formulation using reference-scaled average bioequivalence analysis, the point estimates were within the $0.80-1.25$ range, and the upper one-sided 95\% CIs of scaled average bioequivalence scale metrics were less than 0 . The point estimates and associated $90 \%$ CIs for the ratios comparing $C_{\text {max }}, \mathrm{AUC}_{\text {last }}$, and AUC of clopidogrel following administration of the test versus reference formulation based on average bioequivalence scale analysis were within the acceptable range of 0.80-1.25 for bioequivalence.
Chinese subjects. a Linear model-clopidogrel; b semilogarithmic scale; c linear model-SR26334; d semilogarithmic scale-SR26334

\section{Safety}

There were no serious adverse events (SAEs) or adverse events of special interest (AESIs) reported. There were no reports of permanent treatment discontinuation due to treatment emergent adverse events (TEAEs). TEAEs were observed in 4 subjects during the entire study period: in 3 of 170 subjects $(1.8 \%)$ receiving the reference formulation and in 1 of 165 subjects $(0.6 \%)$ receiving the test formulation.

The following TEAEs until end of study (EOS) visit were reported. Subjects receiving the reference formulation: One subject experienced nausea $(0.6 \%)$ and retching $(0.6 \%)$ during the second period, one subject $(0.6 \%)$ experienced pyrexia during the third period, and one subject $(0.6 \%)$ experienced confusional state before investigational medicinal product during the first period and this TEAE was considered not related to the treatment by the investigator. Subjects receiving the test formulation: One subject $(0.6 \%)$ experienced confusional state due to hemophobia before investigational medicinal product during the first treatment and it was considered not related to the 
Table 2 Pharmacokinetic parameters of acetylsalicylic acid and clopidogrel for test and reference formulations

\begin{tabular}{|c|c|c|c|c|}
\hline \multirow[t]{2}{*}{ Parameters } & \multicolumn{4}{|l|}{ Acetylsalicylic acid } \\
\hline & $\overline{\text { Combination }}$ & Pooled & Replicate 1 & Replicate 2 \\
\hline \multicolumn{5}{|l|}{$C_{\max }$} \\
\hline Number & 162 & 332 & 168 & 164 \\
\hline Mean (SD) (ng/mL) & $790(504)$ & $688(438)$ & $679(428)$ & $698(449)$ \\
\hline Geometric mean $(\mathrm{ng} / \mathrm{mL})$ & 635 & 532 & 533 & 531 \\
\hline $\mathrm{CV}(\%)$ & 64 & 64 & 63 & 64 \\
\hline \multicolumn{5}{|l|}{$\mathrm{AUC}_{\text {last }}$} \\
\hline Number & 162 & 332 & 168 & 164 \\
\hline Mean (SD) (ng h/mL) & $967(402)$ & $901(395)$ & $881(375)$ & $921(416)$ \\
\hline Geometric mean $(\mathrm{ng} \mathrm{h} / \mathrm{mL})$ & 877 & 802 & 794 & 811 \\
\hline CV (\%) & 42 & 44 & 43 & 45 \\
\hline \multicolumn{5}{|l|}{ AUC } \\
\hline Number & 134 & 259 & 132 & 127 \\
\hline Mean (SD) (ng h/mL) & $1020(397)$ & $986(386)$ & $942(369)$ & $1030(399)$ \\
\hline Geometric mean $(\mathrm{ng} \mathrm{h} / \mathrm{mL})$ & 942 & 903 & 856 & 953 \\
\hline CV (\%) & 39 & 39 & 39 & 39 \\
\hline \multicolumn{5}{|l|}{$T_{\max }$} \\
\hline Number & 162 & 332 & 168 & 164 \\
\hline Median (min, max) (h) & $5.00(3.00-12.00)$ & $5.50(2.00-12.00)$ & $5.50(2.00-12.00)$ & $5.50(3.00-12.00)$ \\
\hline \multicolumn{5}{|l|}{$t_{\text {last }}$} \\
\hline Number & 162 & 332 & 168 & 164 \\
\hline Median $(\min , \max )(\mathrm{h})$ & $8.00(5.00-12.03)$ & $8.00(5.50-16.00)$ & $8.00(5.50-16.00)$ & $8.00(6.00-12.00)$ \\
\hline \multicolumn{5}{|l|}{$t_{1 / 2 z}$} \\
\hline Number & 134 & 261 & 133 & 128 \\
\hline Mean (SD) (h) & $0.455(0.165)$ & $0.471(0.312)$ & $0.484(0.328)$ & $0.457(0.294)$ \\
\hline Geometric mean $(\mathrm{h})$ & 0.433 & 0.428 & 0.436 & 0.421 \\
\hline \multirow[t]{2}{*}{$\mathrm{CV}(\%)$} & 36 & 66 & 68 & 64 \\
\hline & \multicolumn{4}{|l|}{ Clopidogrel } \\
\hline \multicolumn{5}{|l|}{$\overline{C_{\max }}$} \\
\hline Number & 165 & 334 & 170 & 164 \\
\hline Mean $(\mathrm{SD})(\mathrm{pg} / \mathrm{mL})$ & $2390(3940)$ & $2130(3610)$ & $2120(3830)$ & $2150(3390)$ \\
\hline Geometric mean $(\mathrm{pg} / \mathrm{mL})$ & 1140 & 1040 & 991 & 1090 \\
\hline
\end{tabular}


Table 2 continued

\begin{tabular}{|c|c|c|c|c|}
\hline & Clopidogrel & & & \\
\hline CV (\%) & 164 & 170 & 181 & 158 \\
\hline $\mathrm{AUC}_{\text {last }}$ & & & & \\
\hline Number & 165 & 334 & 170 & 164 \\
\hline Mean (SD) $(\mathrm{pg} \mathrm{h} / \mathrm{mL})$ & $2810(4650)$ & $2980(4910)$ & $2890(4960)$ & $3080(4880)$ \\
\hline Geometric mean $(\mathrm{pg} \mathrm{h} / \mathrm{mL})$ & 1500 & 1610 & 1530 & 1690 \\
\hline CV (\%) & 166 & 165 & 172 & 159 \\
\hline AUC & & & & \\
\hline Number & 154 & 281 & 146 & 135 \\
\hline Mean $(\mathrm{SD})(\mathrm{pg} \mathrm{h} / \mathrm{mL})$ & $3020(4840)$ & $3240(5310)$ & $3060(5310)$ & $3430(5330)$ \\
\hline Geometric mean $(\mathrm{pg} \mathrm{h} / \mathrm{mL})$ & 1670 & 1750 & 1620 & 1910 \\
\hline $\mathrm{CV}(\%)$ & 160 & 164 & 173 & 155 \\
\hline$T_{\max }$ & & & & \\
\hline Number & 165 & 334 & 170 & 164 \\
\hline Median (min, max) (h) & $0.50(0.17-3.05)$ & $0.75(0.17-12.00)$ & $0.75(0.17-12.00)$ & $0.75(0.17-4.00)$ \\
\hline$t_{\text {last }}$ & & & & \\
\hline Number & 165 & 334 & 170 & 164 \\
\hline Median (min, max) (h) & $16.00(4.00-24.02)$ & $24.00(3.00-24.05)$ & $24.00(6.00-24.03)$ & $24.00(3.00-24.05)$ \\
\hline$t_{1 / 2 z}$ & & & & \\
\hline Number & 155 & 283 & 147 & 136 \\
\hline Mean (SD) $(\mathrm{h})$ & $4.94(1.97)$ & $5.88(2.96)$ & $5.94(3.31)$ & $5.81(2.53)$ \\
\hline Geometric mean $(\mathrm{h})$ & 4.51 & 5.18 & 5.16 & 5.20 \\
\hline
\end{tabular}

$A U C$ area under the plasma concentration versus time, $C_{\text {max }}$ maximum plasma concentration observed, $A U C_{\text {last }}$ area under the plasma concentration versus time curve calculated using the trapezoidal method from time zero to the real time, $T_{\text {max }}$ time to reach $C_{\max }$ curve, $t_{\text {last }}$ time corresponding to the last concentration above the limit of quantification, $t_{1 / 2 z}$ terminal half-life associated with the terminal slope, $C V$ coefficient of variation

treatment by the investigator. All TEAEs were mild in intensity and eventually resolved.

\section{DISCUSSION}

This is the first study to our knowledge that compares the pharmacokinetics of a fixed-dose combination of acetylsalicylic acid and clopidogrel to that of the coadministered individual formulations in the Chinese population, as well as the first to assess the bioequivalence of highly variable enteric acetylsalicylic acid using the reference-scaled average bioequivalence approach. The analysis of the pharmacokinetic parameters in our study confirmed the bioequivalence of the fixed-dose combination to that of their individual formulations in healthy Chinese subjects. Thus, it is expected that the fixed-dose combination would provide the same therapeutic effect as administration of the 
Table 3 Determination of bioequivalence test method for acetylsalicylic acid

\begin{tabular}{cccc}
\hline Parameter & $\boldsymbol{S}_{\mathbf{W R}}$ & $\mathbf{C V}_{\mathbf{W R}}$ & $\begin{array}{l}\text { Reference scaled average } \\
\text { bioequivalence } \\
\text { conclusion }\end{array}$ \\
\hline $\log \left(C_{\max }\right)$ & 0.721 & 0.826 & $\begin{array}{c}\mathrm{RSABE} \text { applicable } \\
\left(S_{\mathrm{WR}} \geq 0.294\right)\end{array}$ \\
$\log$ & 0.472 & 0.500 & $\begin{array}{c}\mathrm{RSABE} \text { applicable } \\
\left(S_{\mathrm{WR}} \geq 0.294\right)\end{array}$ \\
$\left(\mathrm{AUC} \mathrm{Clast}_{\mathrm{l}}\right)$ & & & $\mathrm{RSABE}$ applicable \\
$\log (\mathrm{AUC})$ & 0.406 & 0.424 & $\left(S_{\mathrm{WR}} \geq 0.294\right)$ \\
\hline
\end{tabular}

$C V_{W R}$ calculated for raw pharmacokinetic parameters, $A U C$ area under the plasma concentration versus time, $C_{\max }$ maximum plasma concentration observed, $A U C_{\text {last }}$ area under the plasma concentration versus time curve calculated using the trapezoidal method from time zero to the real time

two separate individual drugs and can be prescribed in Chinese patients who already take two single drugs.

So far, four bioequivalence studies have been reported in healthy volunteers, all of which used the average bioequivalence approach for determining bioequivalence of their formulation. The two studies with Japanese subjects did not meet the bioequivalence standard for enteric-coated acetylsalicylic acid, possibly because of the high variability of the drug
$[12,13]$. In addition, a study with Korean subjects also did not meet the bioequivalence requirement [15]. The fourth study, performed with Korean subjects, established bioequivalence in Korean men using the average bioequivalence approach, though the data reported the pharmacokinetics of acetylsalicylic acid with high intrasubject variability [16].

One important reason for the failure to demonstrated bioequivalence in these studies might be due to insufficient sample sizes using the average bioequivalence scale approach for drugs with high intrasubject coefficient of variation, considering that the geometric mean ratios of the test to reference formulation were within 0.8-1.25 (though the two-sided 90\% CI for the geometric mean did not meet the bioequivalence criterion), and all studies adopted the two-sequence, two-period crossover study designs and similar bioanalytic methods. Among the different methods of establishing bioequivalence, the average bioequivalence scale approach is the most commonly accepted method of statistical analysis. The average bioequivalence scale method uses a fixed, preset regulatory threshold to define bioequivalence, but does not account for the intrasubject coefficient of variation. Hence, an alternative, an individual bioequivalence (IBE) approach had been proposed to account for high intrasubject variability. Although the IBE method accounts for intrasubject variability, it uses

Table 4 Formulation effect on $C_{\max }, \mathrm{AUC}_{\text {last }}$, and AUC for acetylsalicylic acid in test versus reference

\begin{tabular}{lllll}
\hline Parameter & Estimate & $\begin{array}{l}\text { Acetylsalicylic acid } \\
\end{array}$ & & \multicolumn{2}{l}{ Clopidogrel $^{*}$} & \\
\cline { 4 - 5 } & & $\mathbf{9 5 \%} \mathbf{C I}$ for $\left(\boldsymbol{\mu}_{\mathbf{T}}-\boldsymbol{\mu}_{\mathbf{R}}\right)^{\mathbf{2}}-\boldsymbol{\theta} \sigma_{\mathrm{WR}}^{2}$ & Estimate & $\mathbf{9 0 \% ~ C I ~}$ \\
\hline$C_{\max }$ & 1.19 & -0.30 & 1.11 & $1.05-1.18$ \\
AUC $_{\text {last }}$ & 1.09 & -0.14 & 0.95 & $0.91-0.99$ \\
AUC & 1.04 & -0.10 & 0.96 & $0.92-1.00$ \\
\hline
\end{tabular}

$A U C$ area under the plasma concentration versus time, $C_{\text {max }}$ maximum plasma concentration observed, $A U C_{\text {last }}$ area under the plasma concentration versus time curve calculated using the trapezoidal method from time zero to the real time

${ }^{*}$ Point estimates of formulation ratios and upper one-sided $95 \%$ confidence interval for the reference scaled average bioequivalence metrics $\left(S_{\mathrm{WR}} \geq 0.294\right)$; upper 95\% CI for $\left(\mu_{\mathrm{T}}-\mu_{\mathrm{R}}\right)^{2}-\theta \sigma_{\mathrm{WR}}^{2} \theta=\left[\ln (1.25) / \sigma_{0}\right]^{2}$ with $\sigma_{0}=0.250$ corresponding to a regulatory constant

\# Point estimates of formulation ratios with $90 \%$ CIs using average bioequivalence analysis 
predetermined values for the intrasubject variability [17].

Drugs in which the intrasubject variability is at least $30 \%$ of the $C_{\max }$ and/or AUC are referred to as highly variable drugs [18]. The most suitable approach to evaluate the bioequivalence of a highly variable drug is reference-scaled average bioequivalence, for which the acceptance limit of geometric mean ratio should be within $0.8-1.25$ and the acceptance limits could be scaled to the variability of the reference formulation in a replicate trial design [18]. This approach has been recommended by the US Food and Drug Administration (FDA) [19] and can be used when the drug has high intrasubject variability. With the reference-scaled average bioequivalence method, a three-period, threesequence crossover or a two-period, four-sequence crossover study design for evaluating bioequivalence of highly variable drugs is recommended [20].

As a result of high intrasubject variability of acetylsalicylic acid reported in various studies [21-23] and in order to minimize the sample size and also to account for intrasubject variability, the reference-scaled average bioequivalence approach was proposed in our study for assessing the bioequivalence of acetylsalicylic acid within the fixed-dose combination. In the study, the sufficient sample size was calculated on the basis of the pooled intrasubject variability of the individual drugs with an overall power of $90 \%$. A $20 \%$ dropout rate was also considered to ensure that pharmacokinetic data from a sufficient number of subjects were obtained from the study.

The reference-scaled average bioequivalence approach was successfully applied for the demonstration of bioequivalence in the study. The $S_{\mathrm{WR}}$ of log-transformed $C_{\max }, \mathrm{AUC}$, and $\mathrm{AUC}_{\text {last }}$ for acetylsalicylic acid was at least 0.294. The point estimates and $95 \%$ CIs for AUC, $C_{\max }$, and $\mathrm{AUC}_{\text {last }}$ for acetylsalicylic acid determined by reference-scaled average bioequivalence were within the acceptable range, thereby indicating the bioequivalence of fixed-dose combination. The pharmacokinetic parameters for both enteric-coated acetylsalicylic acid and clopidogrel were also comparable with previous studies $[12,13]$.
However our study is not without limitations. First, we enrolled healthy subjects in the study as this decreases the potential for concomitant medications and the presence of underlying disease, which may confound the results of the study. However, in real-world clinical practice, the pharmacokinetics might be different in other targeted populations, especially in elderly patients, or after other dosage regimens. Second, although standard meals were provided during days of hospitalization in the study, the calories for a given type of meal may have slightly varied across the three periods, which may add to the intrasubject variation for the enteric formulation. Third, this study was performed under fasting conditions and an additional study would be required to evaluate bioequivalence results in fed conditions.

\section{CONCLUSIONS}

To our knowledge, this is the first article to demonstrate the bioequivalence of the fixeddose combination with separate formulations in healthy Chinese volunteers under fasting condition. The reference-scaled average bioequivalence approach was successfully applied to evaluate the bioequivalence of acetylsalicylic acid in Chinese male and female volunteers under fasting conditions. This study found that the test (Co-Plavix) and reference formulations were bioequivalent.

\section{ACKNOWLEDGEMENTS}

The authors acknowledge Dan Zheng, Medical Communication, Sanofi, China, for publication process coordination, Xin Xie, Shan Wang, and Shanshan Yang for their contribution to the implementation of clinical trials, Yucheng Sheng, for the protocol design, Jing Jin, R\&D, Sanofi, China for statistical supervision, Rui Chen and Jun $\mathrm{Lu}, \mathrm{R} \& \mathrm{D}$, Sanofi, China for study oversight, Yongzhen $\mathrm{Gu}, \mathrm{R} \& \mathrm{D}$, Sanofi, China for study conduct, and Yingying Han, Jingjing Wei, and Yanqiu Huang for CSR writing support. In addition, the authors would like to thank all the participants of the study. 
Funding. This study, the journal's rapid service and open access fees were sponsored by Sanofi.

Authorship. All named authors meet the International Committee of Medical Journal Editors (ICMJE) criteria for authorship for this article, take responsibility for the integrity of the work as a whole, and have given their approval for this version to be published.

Authorship Contributions. Conceptualization: Jie Hou, Lu Wang, Jeffrey E. Ming, Huiqiu Yin. Data acquisition: Jie Hou, Lu Wang. Data analysis or interpretation: Jie Hou, Lu Wang, Yuijing Di, Tinging Guo, Jeffrey E. Ming, Fangyuan Kong, Linlin Zhang, Fang Xie, Na Yang, Chuan Ping, Yi Li. Drafting: Jeffrey E. Ming, Fangyuan Kong, Lu Wang. Critical Revision: Jie Hou, Yuijing Di, Tinging Guo, Jeffrey E. Ming, Fangyuan Kong, Huiqiu Yin, Linlin Zhang, Fang Xie, Na Yang, Chuan Ping, Yi Li. Final Approval: Jie Hou, Lu Wang, Yuijing Di, Tinging Guo, Jeffrey E. Ming, Fangyuan Kong, Huiqiu Yin, Linlin Zhang, Fang Xie, Na Yang, Chuan Ping, Yi Li. Accountable for accuracy and integrity: Jie Hou, Lu Wang, Yuijing Di, Tinging Guo, Jeffrey E. Ming, Fangyuan Kong, Huiqiu Yin, Linlin Zhang, Fang Xie, Na Yang, Chuan Ping, Yi Li.

Medical Writing and Editorial Assistance. The authors acknowledge Anwesha Mandal and Dr. G. Kaushik Subramanian, Indegene pvt. Ltd, India, for medical writing support.

Disclosures. Jeffrey E. Ming is an employee of Sanofi and may hold stock and/or stock options in the company. Fangyuan Kong, Huiqiu Yin, Linlin Zhang, Fang Xie, Na Yangand, and Yi Li are employees of Sanofi. Chuan Ping, a former employee of Sanofi, now is an employee of Novartis. Jie Hou, Lu Wang, Yuijing Di, and Tingting Guo have nothing to disclose.

Compliance with Ethics Guidelines. The study protocol and informed consent to participate were reviewed and approved by the Institutional Review Board (IRB) of PKUCare Luzhong Hospital. The study was conducted in accordance with consensus ethics principles derived from international ethics guidelines, including the Declaration of Helsinki 1956 and its later amendments, and The International Council for Harmonisation of Technical Requirements for Pharmaceuticals for Human Use guidelines for Good Clinical Practice, and all applicable laws, rules, and regulations.

Data Availability. Qualified researchers may request access to patient-level data and related study documents including the clinical study report, study protocol with any amendments, blank case report form, statistical analysis plan, and data set specifications. Patient-level data will be anonymized, and study documents will be redacted to protect the privacy of trial participants. Further details on Sanofi's data sharing criteria, eligible studies, and process for requesting access can be found at the following website: https://www.clinicalstudydatarequest. com.

Open Access. This article is licensed under a Creative Commons Attribution-NonCommercial 4.0 International License, which permits any non-commercial use, sharing, adaptation, distribution and reproduction in any medium or format, as long as you give appropriate credit to the original author(s) and the source, provide a link to the Creative Commons licence, and indicate if changes were made. The images or other third party material in this article are included in the article's Creative Commons licence, unless indicated otherwise in a credit line to the material. If material is not included in the article's Creative Commons licence and your intended use is not permitted by statutory regulation or exceeds the permitted use, you will need to obtain permission directly from the copyright holder. To view a copy of this licence, visit http:// creativecommons.org/licenses/by-nc/4.0/.

\section{REFERENCES}

1. Layne K, Ferro A. Antiplatelet therapy in acute coronary syndrome. Eur Cardiol. 2017;12:33-7. 
2. Rosenkranz B, Fischer C, Meese CO, Frolich JC. Effects of salicylic and acetylsalicylic acid alone and in combination on platelet aggregation and prostanoid synthesis in man. Br J Clin Pharmacol. 1986;21:309-17.

3. Olesen OV, Linnet K. Contributions of five human cytochrome $\mathrm{P} 450$ isoforms to the $\mathrm{N}$-demethylation of clozapine in vitro at low and high concentrations. J Clin Pharmacol. 2001;41:823-32.

4. Mukherjee D, Fang J, Chetcuti S, Moscucci M, Kline-Rogers E, Eagle KA. Impact of combination evidence-based medical therapy on mortality in patients with acute coronary syndromes. Circulation. 2004;109:745-9.

5. Eagle KA, Kline-Rogers E, Goodman SG, et al. Adherence to evidence-based therapies after discharge for acute coronary syndromes: an ongoing prospective, observational study. Am J Med. 2004;117:73-81.

6. Collet J-P, Aout M, Alantar A, et al. Real-life management of dual antiplatelet therapy interruption: the REGINA survey. Arch Cardiovasc Dis. 2009;102: 697-710.

7. Sabatine MS, Cannon CP, Gibson CM, et al. Addition of clopidogrel to aspirin and fibrinolytic therapy for myocardial infarction with ST-segment elevation. N Engl J Med. 2005;352:1179-89.

8. Sabatine MS, Cannon CP, Gibson CM, et al. Effect of clopidogrel pretreatment before percutaneous coronary intervention in patients with ST-elevation myocardial infarction treated with fibrinolytics: the PCI-CLARITY study. JAMA. 2005;294:1224-32.

9. Yusuf S, Zhao F, Mehta SR, et al. Effects of clopidogrel in addition to aspirin in patients with acute coronary syndromes without ST-segment elevation. N Engl J Med. 2001;345:494-502.

10. Chen ZM, Jiang LX, Chen YP, et al. Addition of clopidogrel to aspirin in 45,852 patients with acute myocardial infarction: randomised placebo-controlled trial. Lancet. 2005;366:1607-21.

11. Haidar SH, Davit B, Chen M-L, et al. Bioequivalence approaches for highly variable drugs and drug products. Pharm Res. 2008;25:237-41.

12. Reexamination report, Pharmaceuticals and Medical Devices Agency [Internet]. 2015 Jul p. 1-29. Available from: https://e-mr.sanofi.co.jp/-/media/ EMS/Conditions/eMR/di/interview/complavin.pdf.

13. Examination report, Pharmaceuticals and Medical Devices Agency, The following are the results of the review by the Pharmaceuticals and Medical Devices Agency for the following drugs that have been submitted for approval. [Internet]. 2013 Aug p. 1-18. Available from: https://e-mr.sanofi.co.jp/-/ media/EMS/Conditions/eMR/di/interview/ complavin.pdf.

14. Abbreviates Study Clinical Study Report. An openlabel, randomized, single-dose, two-sequence crossover relative bioavailability study of a tablet containing $75 \mathrm{mg}$ of clopidogrel and $100 \mathrm{mg}$ of aspirin versus the simultaneous administration of the separate formulations of the two drugs in Japanese healthy male subjects STUDY NUMBER: BDR11360, 2010;1-68 (Unpublished Manuscript).

15. Jung JA, Kim T-E, Kim J-R, et al. The pharmacokinetics and safety of a fixed-dose combination of acetylsalicylic acid and clopidogrel compared with the concurrent administration of acetylsalicylic acid and clopidogrel in healthy subjects: a randomized, open-label, 2-sequence, 2-period, singledose crossover study. Clin Ther. 2013;35:985-94.

16. Choi H-K, Ghim J-L, Shon J, Choi Y-K, Jung JA. Pharmacokinetics and relative bioavailability of fixed-dose combination of clopidogrel and aspirin versus coadministration of individual formulations in healthy Korean men. Drug Des Dev Ther. 2016;10:3493-9.

17. Patnaik RN, Lesko LJ, Chen ML, Williams RL. Individual bioequivalence. Clin Pharmacokinet. 1997;33:1-6.

18. Midha KK, Rawson MJ, Hubbard JW. The bioequivalence of highly variable drugs and drug products. Int J Clin Pharmacol Ther. 2005;43:485-98.

19. Davit BM, Chen M-L, Conner DP, et al. Implementation of a reference-scaled average bioequivalence approach for highly variable generic drug products by the us food and drug administration. AAPS J. 2012;14:915-24.

20. Tothfalusi L, Endrenyi L. An exact procedure for the evaluation of reference-scaled average bioequivalence. AAPS J. 2016;18:476-89.

21. Benedek IH, Joshi AS, Pieniaszek HJ, King S-YP, Kornhauser DM. Variability in the pharmacokinetics and pharmacodynamics of low dose aspirin in healthy male volunteers. J Clin Pharmacol. 1995;35:1181-6.

22. Ferguson AD, Dokainish H, Lakkis N. Aspirin and clopidogrel response variability. Tex Heart Inst J. 2008;35:313-20.

23. Dolores RC, Antunes NJ, Moreno R, Di Vaio P, Magli E. Comparative bioavailability study of two $81 \mathrm{mg}$ coated tablet formulations of acetylsalicylic acid in fasting healthy volunteers. J Bioequiv Bioavailab. 2017;09:477. 